\title{
Multi-scale high order finite different reverse time migration imaging
}

\author{
Xiaodan Zhang", Lei Zhu and Hua Guo
}

\begin{abstract}
At present, the main difficulties in oil and gas exploration are focused on the exploration of the deep region. How to improve the efficiency of imaging on the same accuracy of migration is an urgent problem. The paper puts forward the concept of multi-scale grid for the underground medium based on finite difference time domain (FDTD) of reverse time migration (RTM), which can avoid the oversampling to high speed and hold the sampling density to low-speed layer. The method can shorten the time of RTM with the same accuracy of the traditional RTM imaging. Firstly, devise the multi-scale grid model according to the velocity model; it uses the small-scale grid for the low-speed area and large-scale grid for high-speed area; secondly, calculate the value of each point of the wave field on multi-scale model, especially the points of transition zone; finally, image the underground media according to the RTM imaging condition. The experimental results show that under the condition of the same simulation order, the multi-scale RTM imaging computational efficiency can be promoted by $25.05 \%$ average within this paper.
\end{abstract}

Keywords: Multi-scale, FDTD, RTM imaging, Oversampling, Computational efficiency

\section{Introduction}

In recent years, along with the oil and gas, reservoirs of traditional and simple structure have been mined gradually; the discovery of mineral resources becomes more difficulty and it makes the resources which can be protected and properly used without any way. The exploration is facing the problem of big tilt angle, buried deep, and the drastic change of the medium velocity in horizontal and vertical [1]. So, it is paid more and more attention on how to improve the efficiency and accuracy of migration imaging algorithm. On the one hand, the complexity of the algorithm is increased while the imaging precision improves, which can increase the calculation amount and decrease the computational efficiency. On the other hand, it will inevitably sacrifice migration accuracy while improving the algorithm; it seems to be irreconcilable contradictions of computational efficiency and accuracy.

RTM is based on the exact wave equation rather than its approximation [2]; the time extrapolation is used instead of the depth extrapolation, so it has a good precision. It is not limited by the inclination of

\footnotetext{
* Correspondence: 18192823070@163.com

College of Electronic and Information, Xi'an Polytechnic University, No. 19 Jinhua South Road, Xi'an 710048, China
}

the underground structure and the change of the lateral velocity of the medium, and even can the image of the rotary wave [3-6]. When the finite difference method is performed for RTM imaging, the selection of the difference order directly affects the imaging precision and the amount of calculation [7-9], and the higher the differential order, the more the imaging is accurate, but the amount of RTM is multiplied by the forward and reverse recursion of the wave field $[10,11]$. It makes the computer time-consuming, and the storage demand of the full wave field is large. It seriously restricts RTM in the field. In order to solve the above problems, the predecessors have researched related topics. Reference [12] proposes graphics processing unit (GPU) parallel strategy is used to improve the computing efficiency for RTM; this method starts with the hardware and solves the problem of low computing efficiency. Reference [13] proposes the pseudo spectral method which is used to solve the problem of calculating the spatial derivative, thus improving the computational efficiency of the algorithm. Reference [14] combines the high-order finite difference and boundary conditions to improve the accuracy of the algorithm. Reference [15] used the combination of high calculation efficiency of 
Kirchhoff integral method of ray shift and reverse time migration to improve the efficiency and accuracy. Reference [16-18] proposes an efficient boundary storage strategy of optimizing the random boundary condition and absorbing boundary condition, which can reduce the memory requirement. Reference [19] proposes a reverse time migration method based on the cloud computing to improve the computational efficiency of the algorithm. Reference [20] improves the efficiency of the algorithm from the view of coding and low-order efficiency. The previous work has laid a solid foundation for the subsequent research. In this paper, the RTM algorithm is studied in order to achieve a balance between the improving computing efficiency and the imaging precision. In the discrete analysis of the underground medium, the grid of the same size for the whole model often causes oversampling in the high-speed layer area when the grid is suit for low velocity; if the large grid is used for the whole model, then it can make the low velocity layer and thin layer imaging not clear. So, the paper starts from this problem and designs to use a small-scale mesh in the low velocity layer to make fine imaging, and the large-scale grids are used in the high-velocity layer to avoid oversampling and the amount of computation and memory occupancy also can be reduced. The method can improve the comprehensive efficiency for RTM while the lateral resolution of the migration imaging results is not affected. The experimental results verify the effectiveness of the algorithm in the paper.

\section{Method of multi-scale RTM imaging}

The traditional RTM imaging is performed by a high-order FDTD scheme. The two-dimensional full wave equation is taken as an example, and it can be expressed in Eq. 1 as follows:

$$
\frac{\partial^{2} U}{\partial x^{2}}+\frac{\partial^{2} U}{\partial z^{2}}=\frac{1}{v^{2}} \frac{\partial^{2} U}{\partial t^{2}}+S
$$

In which, $U$ is the wave field function $u(x, z, t), v$ is the medium velocity function $v(x, z), S$ is the source function $s(x, z, t), x, z$ are the space coordinate components, $x$ is the horizontal direction, $z$ is the vertical direction, $t$ is the time direction. For the mathematical equation, if $u(x, z, t)$ is a solution of the wave in Eq. 1, then the $u\left(x, z, t-t_{0}\right)$ is also the solution of the wave in Eq. 1. It is also said that everything in physics can only change with the increase of time, but in mathematics, the time is reversible. Using this principle, the RTM imaging can be realized. The Taylor series is employed in Eq. 1, the $2 \mathrm{~N}$ orders in space and 2 orders in time of the wave equation difference scheme is shown in Eq. 2:

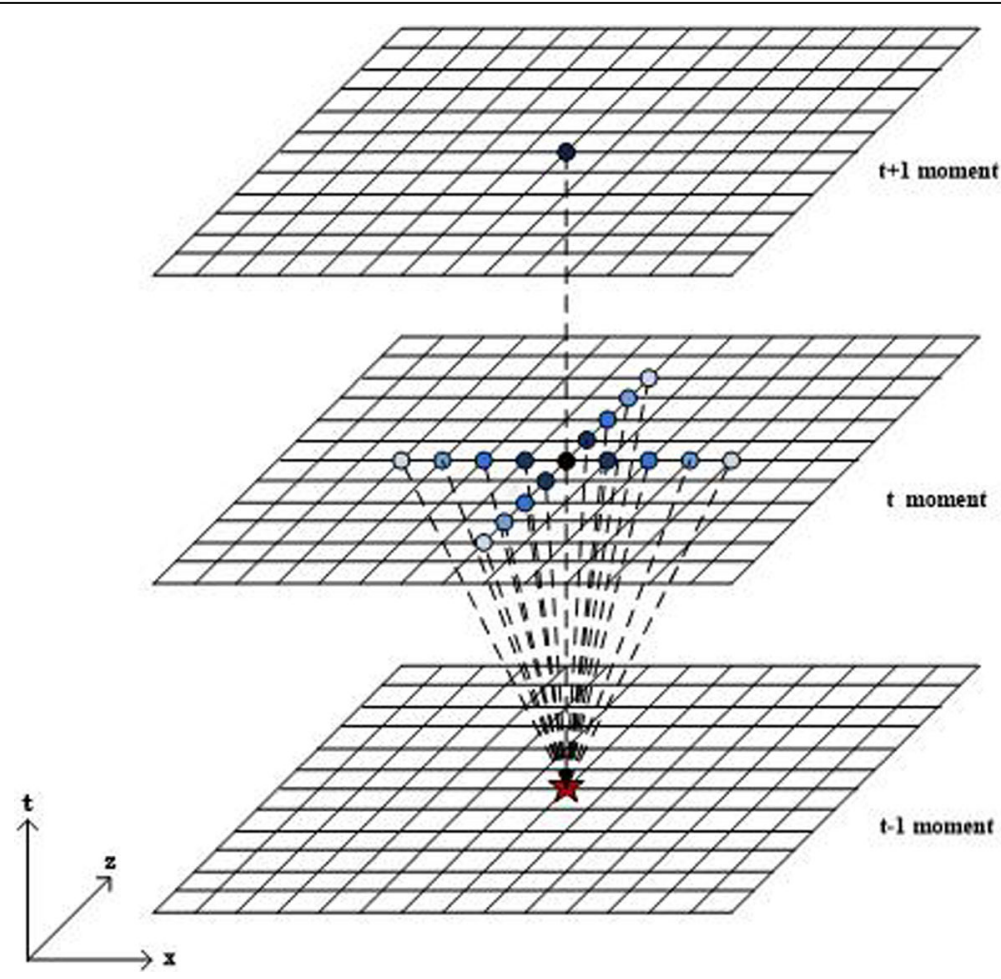

Fig. 1 The 18-point (two orders in the time domain and eight orders in the space domain) format of RTM 


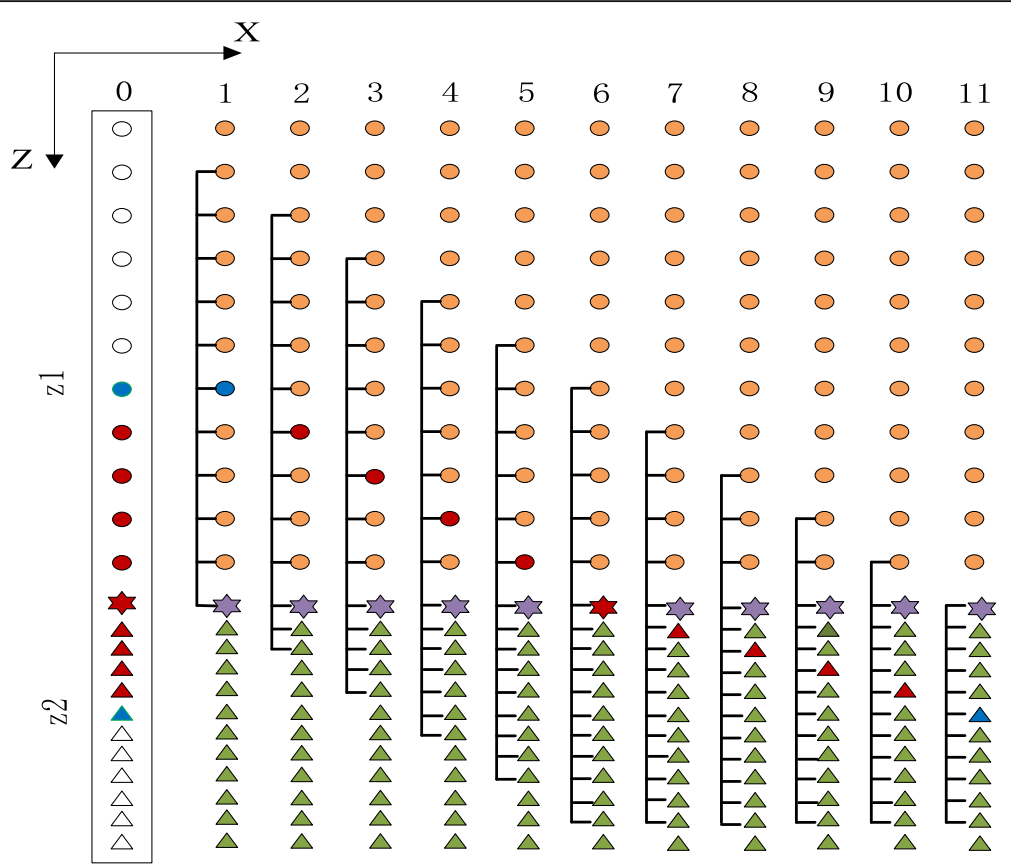

Fig. 2 The deduction wave field of the differential points on transitional zone

$$
\begin{aligned}
u(i, j, k-1) & =2 u(i, j, k)-u(i, j, k+1) \\
& +\frac{\Delta t^{2} V^{2}}{\Delta x^{2}} \sum_{n=1}^{N} C_{n}[u(i+n, j, k)-2 u(i, j, k) \\
& +u(i-n, j, k)]+\frac{\Delta t^{2} V^{2}}{\Delta z^{2}} \sum_{n=1}^{N} C_{n}[u(i, j+n, k) \\
& -2 u(i, j, k)+u(i, j-n, k)] \\
& =2 u(i, j, k)-u(i, j, k+1)+A x \cdot B x+A z \cdot B z
\end{aligned}
$$

In which $C_{n}$ is the difference coefficient and its value can be obtained by Taylor series expansion, and $A x, A z$, $B x, B z$ are shown as follows:

$$
\begin{aligned}
& A x=\frac{V^{2} \Delta t^{2}}{\Delta x^{2}}, A z=\frac{V^{2} \Delta t^{2}}{\Delta z^{2}}, \\
& B x=\sum_{n=1}^{2 N} C_{n}[u(i+n, j, k)-2 u(i, j, k)+u(i-n, j, k)], \\
& B z=\sum_{n=1}^{2 N} C_{n}[u(i, j+n, k)-2 u(i, j, k)+u(i, j-n, k)] .
\end{aligned}
$$

RTM is the convolution imaging of the forward and backward of the wave field; the forward and the backward are reciprocal inverse process. Figure 1 is the 18-point (two orders in the time domain and eight orders in the space domain) format of RTM imaging. The red pentagram on the $t-1$ moment plane in Fig. 1 is the unsolved point which can be calculated from the 17 points on the $t$ moment plane and 1 point on the $t+1$ moment plane is needed as support. The gradient color of the 17 points on the $t$ moment plane shows the degree of association for the unsolved point, and the darker the color, the greater the degree of association is; its value is determined by the difference coefficient.

For a geological model with no dramatic change in lateral velocity, it only needs to consider its multi-scale design in the longitudinal direction, low speed layer using a small step mesh, and big step in high velocity, on the premise of ensuring the imaging accuracy, the amount of calculation is reduced effectively and the computational efficiency is improved. In the calculation of $2 N$ orders in space, the transitional zone is the $2 N-1$ points which is centrosymmetric with the velocity interface; it is found that the changes of the formulas for the transitional zone are concerned with the $A z, B z$ in Eq. 2. Taking the 2 orders in time domain and the 10 orders in space domain as an example, the upper layer uses sparse grid for high-velocity layer and the lower layer uses dense meshes for the low velocity layer, and the sparse grid spacing is 2 times of dense grid spacing. Under this 
condition, it is found that the crux of the wave field value in the transitional zone is the number of the difference point of the 11 points on $t$ time section of $Z$ direction. Figure 2 is the transitional zone of $t$ time on $(x, z)$ plane which is from high-velocity layer to low-velocity layer.

It can be seen from the Fig. 2 that the nine points of red color between $Z 1$ and $Z 2$ on zero line are the transitional zone, the small step length sampling in low-velocity layer which is represented by a triangle and two times of the small step in high-velocity layer which is represented by a circle, the hexagons are the velocity interface. It is supposed that each point in the $X$ direction is at a consistent rate or a little change, so it takes sample with a fixed step length in the $X$ direction, and it can use traditional RTM to compute the wave field values.

The multi-scale FDTD of forward wave field on transitional zone is shown as follows:

The first column has not entered the transition zone, the wave field value of the black point is shown in Eq. 3:

$$
\begin{aligned}
u_{i, j}^{k-1}=2 u_{i, j}^{k} & +u_{i, j}^{k+1}+\frac{V^{2} \Delta t^{2}}{\Delta z^{2}}\left\{a_{5}\left[u_{i, j+5}^{k}+u_{i, j-5}^{k}\right]\right. \\
& +a_{4}\left[u_{i, j+4}^{k}+u_{i, j-4}^{k}\right]+a_{3}\left[u_{i, j+3}^{k}+u_{i, j-3}^{k}\right] \\
& +a_{2}\left[u_{i, j+2}^{k}+u_{i, j-2}^{k}\right]+a_{1}\left[u_{i, j+1}^{k}+u_{i, j-1}^{k}\right] \\
& \left.+a_{0} u_{i, j}^{k}\right\}+\frac{V^{2} \Delta t^{2}}{\Delta x^{2}}\left[\sum_{n=1}^{5} g_{k}\left(u_{i+n, j}^{k}+u_{i-n, j}^{k}-2 u_{i, j}^{k}\right)\right]
\end{aligned}
$$

In which $a_{i}(i=0,1, \cdots 5)$ is the difference coefficient; the number of difference points on both sides of the derivation point is consistent.

The second column begins to enter the transition zone; the difference coefficient and the number of difference point were changed because the step is unequal. $B z$ in Eq. 2 which is at the position of the red point on $t-1$

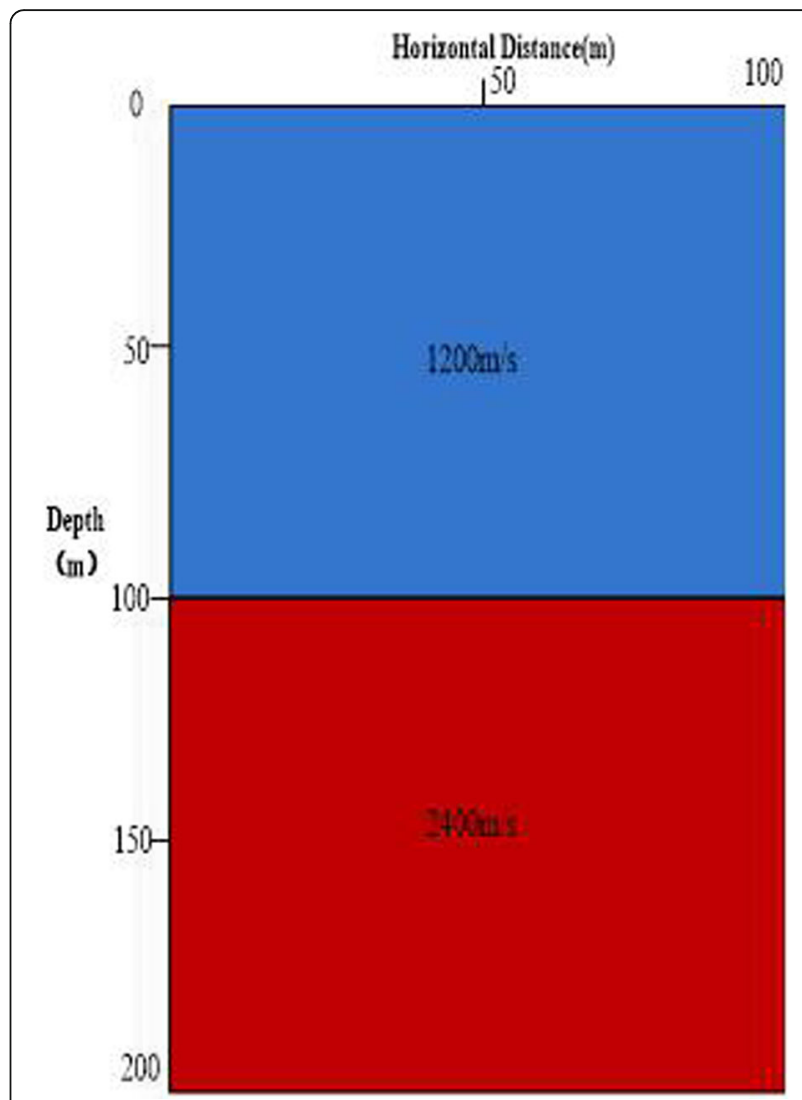

Fig. 3 The velocity model of the flat-layered model

time section is shown in Eq. 4 , and $b_{i}(i=0,1, \cdots, 5)$ is the difference coefficient:

$$
\begin{aligned}
B z= & b_{5}\left[u_{i, j+6}^{k}+u_{i, j-5}^{k}\right]+b_{4}\left[u_{i, j+4}^{k}+u_{i, j-4}^{k}\right] \\
& +b_{3}\left[u_{i, j+3}^{k}+u_{i, j-3}^{k}\right]+b_{2}\left[u_{i, j+2}^{k}+u_{i, j-2}^{k}\right] \\
& +b_{1}\left[u_{i, j+1}^{k}+u_{i, j-1}^{k}\right]+b_{0} u_{i, j}^{k}
\end{aligned}
$$

\begin{tabular}{|c|c|c|c|c|c|c|c|c|c|c|c|c|}
\hline \multirow[b]{2}{*}{$n(1)$} & \multicolumn{2}{|c|}{ First column } & \multicolumn{2}{|c|}{ Second column } & \multicolumn{2}{|c|}{ Third column } & \multicolumn{2}{|c|}{ Fourth column } & \multicolumn{2}{|c|}{ Fifth column } & \multicolumn{2}{|c|}{ Sixth column } \\
\hline & 1 & 1 & 1 & 1 & 1 & 1 & 1 & 1 & 1 & 1 & 2 & 1 \\
\hline$n(2)$ & 2 & 2 & 2 & 2 & 2 & 2 & 2 & 2 & 3 & 2 & 4 & 2 \\
\hline$n(3)$ & 3 & 3 & 3 & 3 & 3 & 3 & 4 & 3 & 5 & 3 & 6 & 3 \\
\hline$n(4)$ & 4 & 4 & 4 & 4 & 5 & 4 & 6 & 4 & 7 & 4 & 8 & 4 \\
\hline$n(5)$ & 5 & 5 & 6 & 5 & 7 & 5 & 8 & 5 & 9 & 5 & 10 & 5 \\
\hline
\end{tabular}

And then, the $B z$ of the red points in the transition zone from the third column to the fifth column are shown as follows:

Table 1 The value of $n(k)$ of 10 order FDTD 


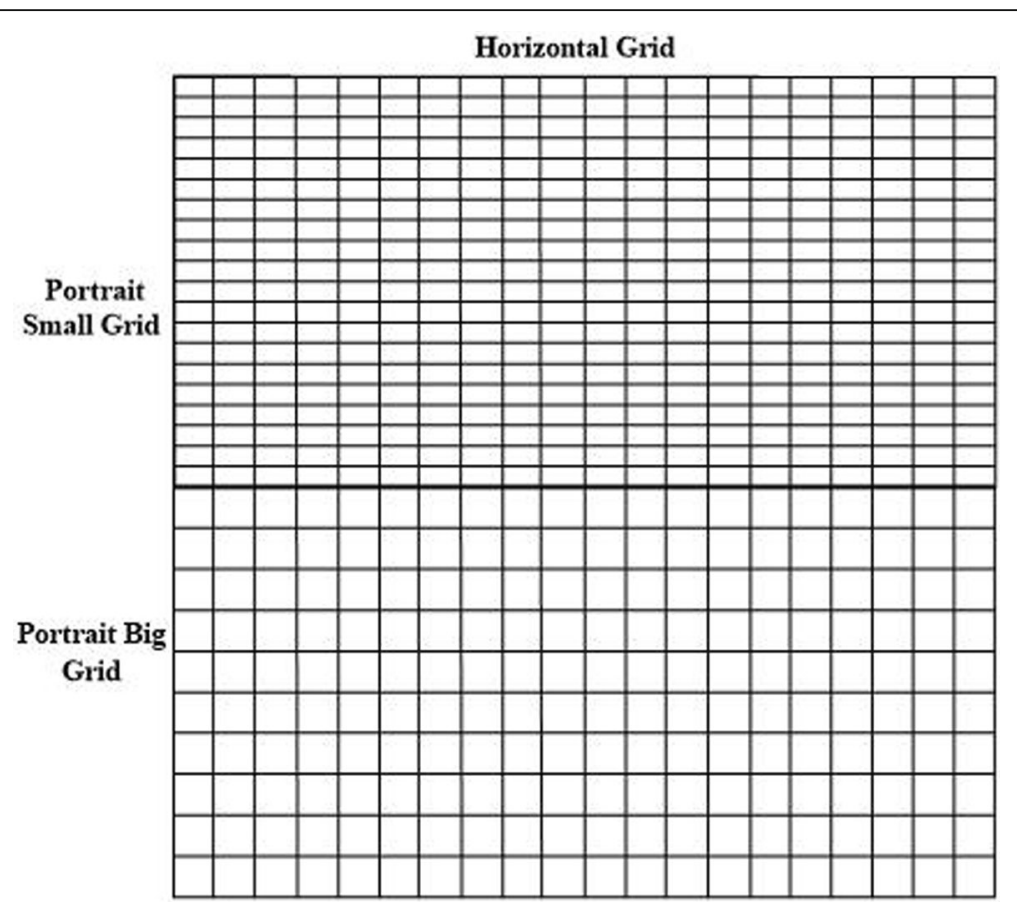

Fig. 4 The multi-scale design of the model

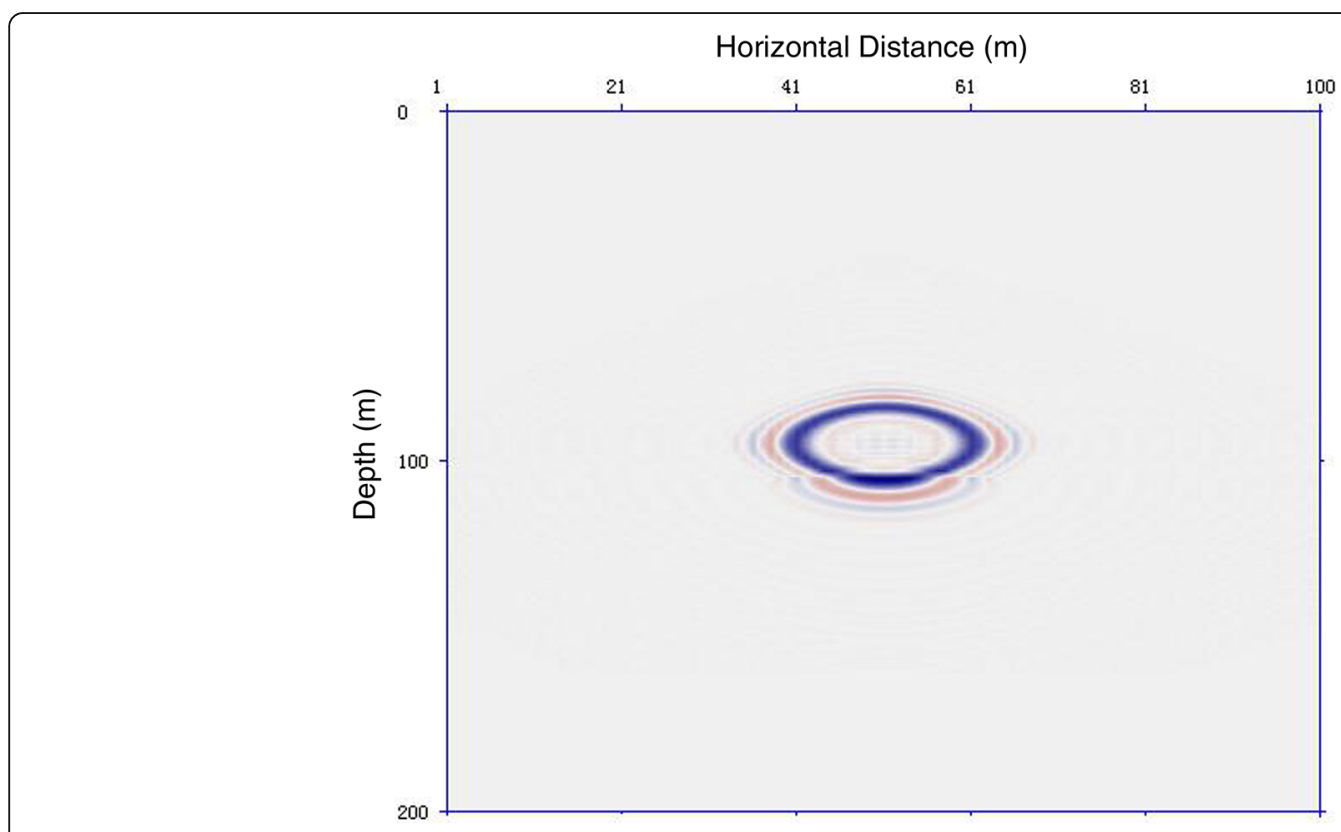

Fig. $5 t=30$ ms snapshot of wave field of multi-scale method 


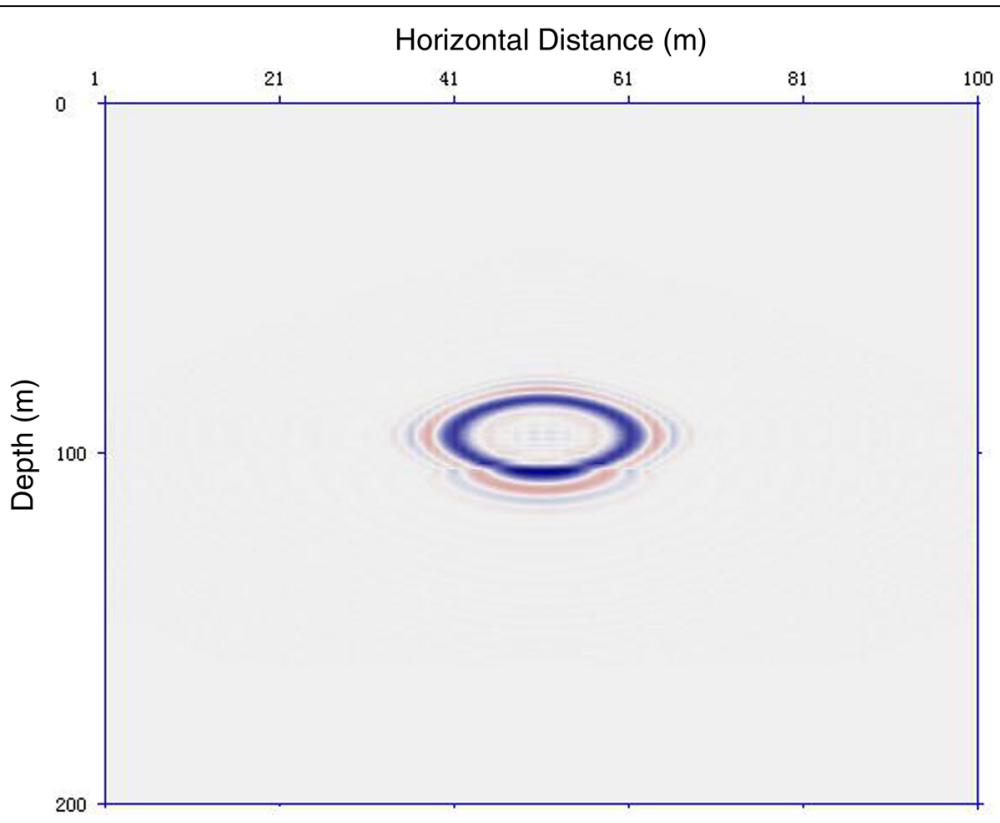

Fig. $6 t=30 \mathrm{~ms}$ snapshot of wave field of traditional method

$$
\begin{aligned}
B z= & c_{5}\left[u_{i, j+7}^{k}+u_{i, j-5}^{k}\right]+c_{4}\left[u_{i, j+5}^{k}+u_{i, j-4}^{k}\right] \\
& +c_{3}\left[u_{i, j+3}^{k}+u_{i, j-3}^{k}\right]+c_{2}\left[u_{i, j+2}^{k}+u_{i, j-2}^{k}\right] \\
& +c_{1}\left[u_{i, j+1}^{k}+u_{i, j-1}^{k}\right]+c_{0} u_{i, j}^{k}
\end{aligned}
$$

$$
\begin{aligned}
B z= & d_{5}\left[u_{i, j+8}^{k}+u_{i, j-5}^{k}\right]+d_{4}\left[u_{i, j+6}^{k}+u_{i, j-4}^{k}\right] \\
& +d_{3}\left[u_{i, j+4}^{k}+u_{i, j-3}^{k}\right]+d_{2}\left[u_{i, j+2}^{k}+u_{i, j-2}^{k}\right] \\
& +d_{1}\left[u_{i, j+1}^{k}+u_{i, j-1}^{k}\right]+d_{0} u_{i, j}^{k}
\end{aligned}
$$

$$
\begin{aligned}
B z= & e_{5}\left[u_{i, j+9}^{k}+u_{i, j-5}^{k}\right]+e_{4}\left[u_{i, j+7}^{k}+u_{i, j-4}^{k}\right] \\
& +e_{3}\left[u_{i, j+5}^{k}+u_{i, j-3}^{k}\right]+e_{2}\left[u_{i, j+3}^{k}+u_{i, j-2}^{k}\right] \\
& +e_{1}\left[u_{i, j+1}^{k}+u_{i, j-1}^{k}\right]+e_{0} u_{i, j}^{k}
\end{aligned}
$$

Table 2 The comparison of calculation time and memory consumption between multi-scale method and traditional method

\begin{tabular}{lll}
\hline Item & Calculation time & Memory consumption \\
\hline Traditional method & $37 \mathrm{~s}$ & $203.49 \mathrm{~kb}$ \\
Multi-scale method & $28 \mathrm{~s}$ & $161.73 \mathrm{~kb}$ \\
Efficiency promotion & $24.32 \%$ & $20.52 \%$ \\
\hline
\end{tabular}

In which $c_{i}, d_{i}, e_{i}(i=0,1, \cdots, 5)$ are the difference coefficients.

The red hexagonal in the sixth column is on the velocity interface, and $A z$ is changed from this column. Based on the change of the grid step, the $A z$ is shown in Eq. 8 and $B z$ is shown in Eq. 9 .

$$
A z=\frac{V^{2} \Delta t_{2}}{\left(\frac{1}{2} \Delta z\right)^{2}}
$$

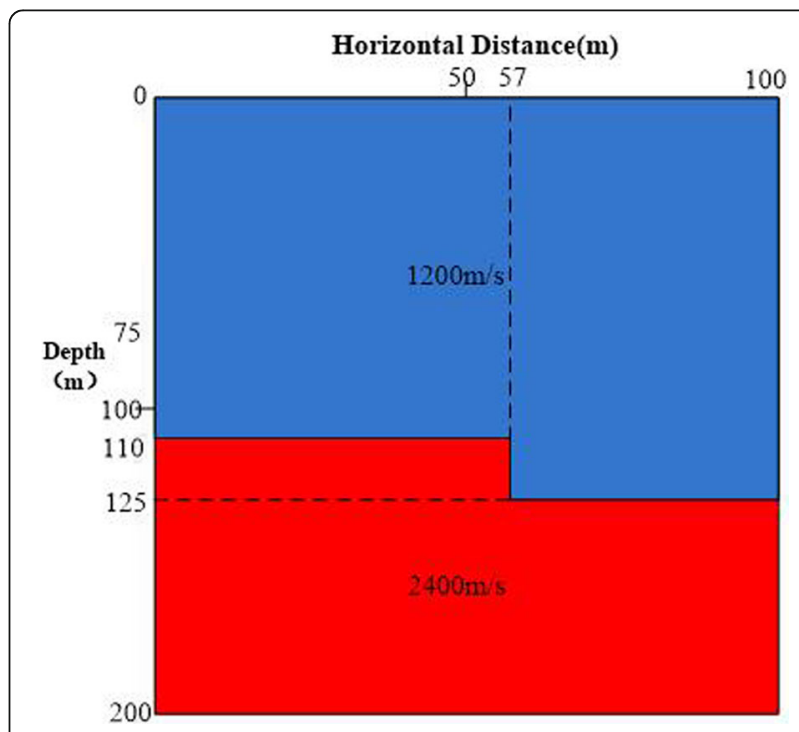

Fig. 7 The velocity model of the step model 


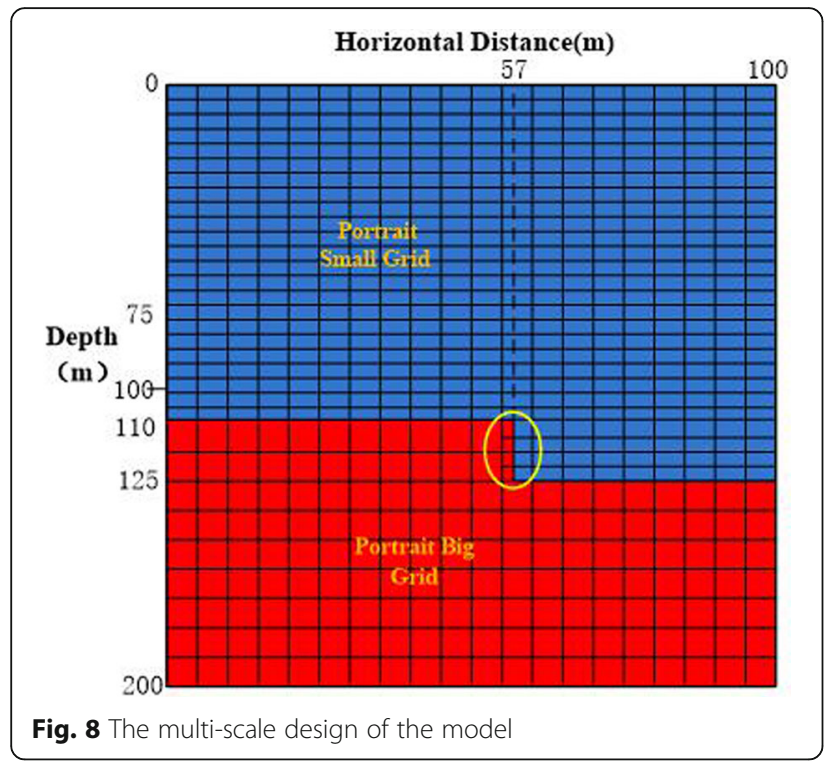

$$
\begin{aligned}
B z= & a_{5}\left[u_{i, j+10}^{k}+u_{i, j-5}^{k}\right]+a_{4}\left[u_{i, j+8}^{k}+u_{i, j-4}^{k}\right] \\
& +a_{3}\left[u_{i, j+6}^{k}+u_{i, j-3}^{k}\right]+a_{2}\left[u_{i, j+4}^{k}+u_{i, j-2}^{k}\right] \\
& +a_{1}\left[u_{i, j+2}^{k}+u_{i, j-1}^{k}\right]+a_{0} u_{i, j}^{k}
\end{aligned}
$$

The $A z$ in Eq. 8 is employed from the seventh column to the tenth column, and $B z$ are shown as follows:

$$
\begin{aligned}
B z= & e_{5}\left[u_{i, j+9}^{k}+u_{i, j-5}^{k}\right]+e_{4}\left[u_{i, j+7}^{k}+u_{i, j-4}^{k}\right] \\
& +e_{3}\left[u_{i, j+5}^{k}+u_{i, j-3}^{k}\right]+e_{2}\left[u_{i, j+3}^{k}+u_{i, j-2}^{k}\right] \\
& +e_{1}\left[u_{i, j+1}^{k}+u_{i, j-1}^{k}\right]+e_{0} u_{i, j}^{k} \\
B z= & d_{5}\left[u_{i, j+8}^{k}+u_{i, j-5}^{k}\right]+d_{4}\left[u_{i, j+6}^{k}+u_{i, j-4}^{k}\right] \\
& +d_{3}\left[u_{i, j+4}^{k}+u_{i, j-3}^{k}\right]+d_{2}\left[u_{i, j+2}^{k}+u_{i, j-2}^{k}\right] \\
& +d_{1}\left[u_{i, j+1}^{k}+u_{i, j-1}^{k}\right]+d_{0} u_{i, j}^{k} \\
& B z= \\
& c_{5}\left[u_{i, j+7}^{k}+u_{i, j-5}^{k}\right]+c_{4}\left[u_{i, j+5}^{k}+u_{i, j-4}^{k}\right] \\
& +c_{3}\left[u_{i, j+3}^{k}+u_{i, j-3}^{k}\right]+c_{2}\left[u_{i, j+2}^{k}+u_{i, j-2}^{k}\right] \\
& +c_{1}\left[u_{i, j+1}^{k}+u_{i, j-1}^{k}\right]+c_{0} u_{i, j}^{k} \\
& +b_{1}\left[u_{i, j+1}^{k}+u_{i, j-1}^{k}\right]+b_{0} u_{i, j}^{k} \\
B z= & b_{5}\left[u_{i, j+6}^{k}+u_{i, j-5}^{k}\right]+b_{4}\left[u_{i, j+4}^{k}+u_{i, j-4}^{k}\right] \\
& +b_{3}\left[u_{i, j+3}^{k}+u_{i, j-3}^{k}\right]+b_{2}\left[u_{i, j+2}^{k}+u_{i, j-2}^{k}\right] \\
& \\
B &
\end{aligned}
$$

It can be observed from the Eqs. 10-13 and Eqs. 7-4 that the wave field formulas of points on the transition

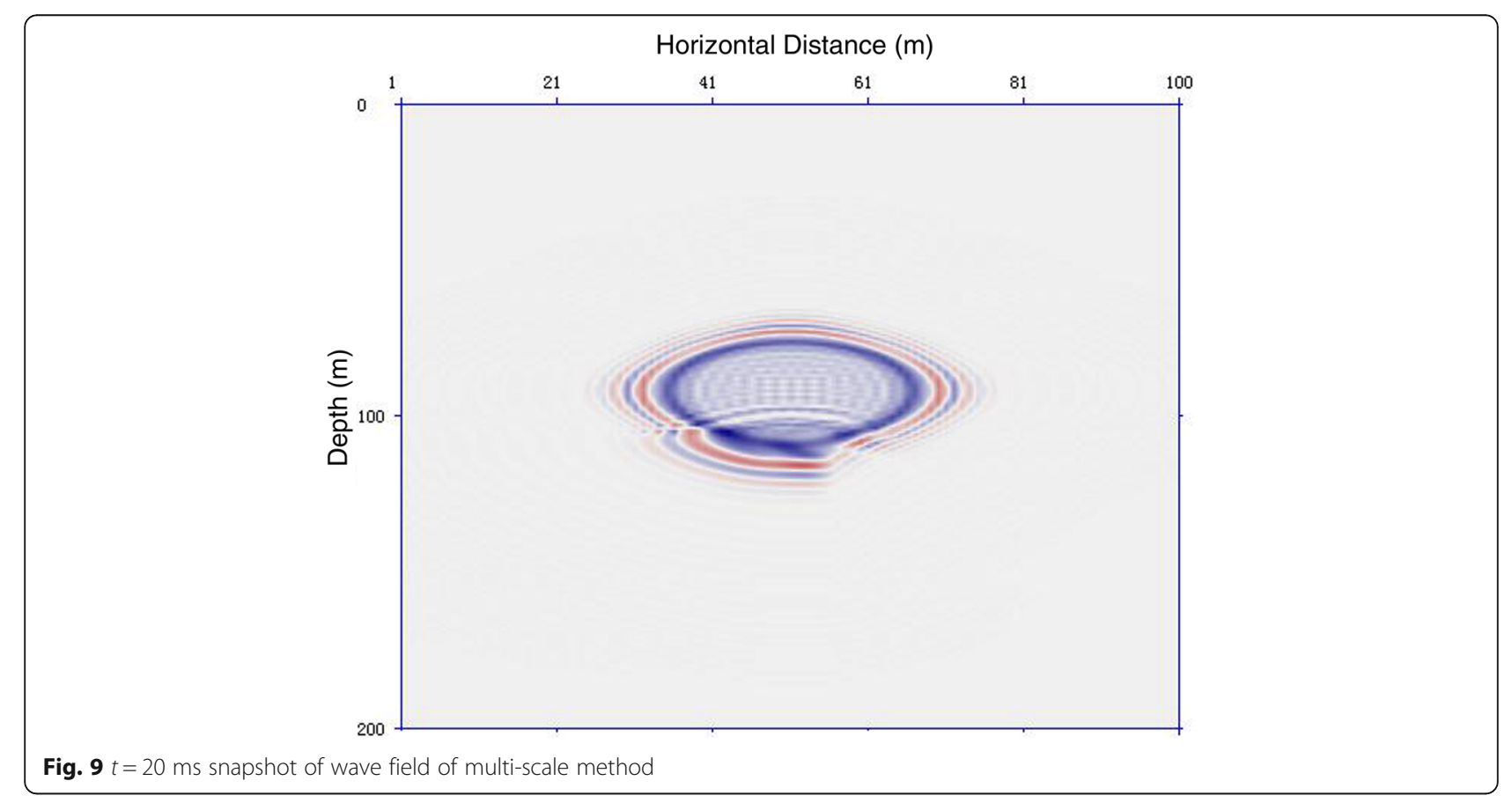




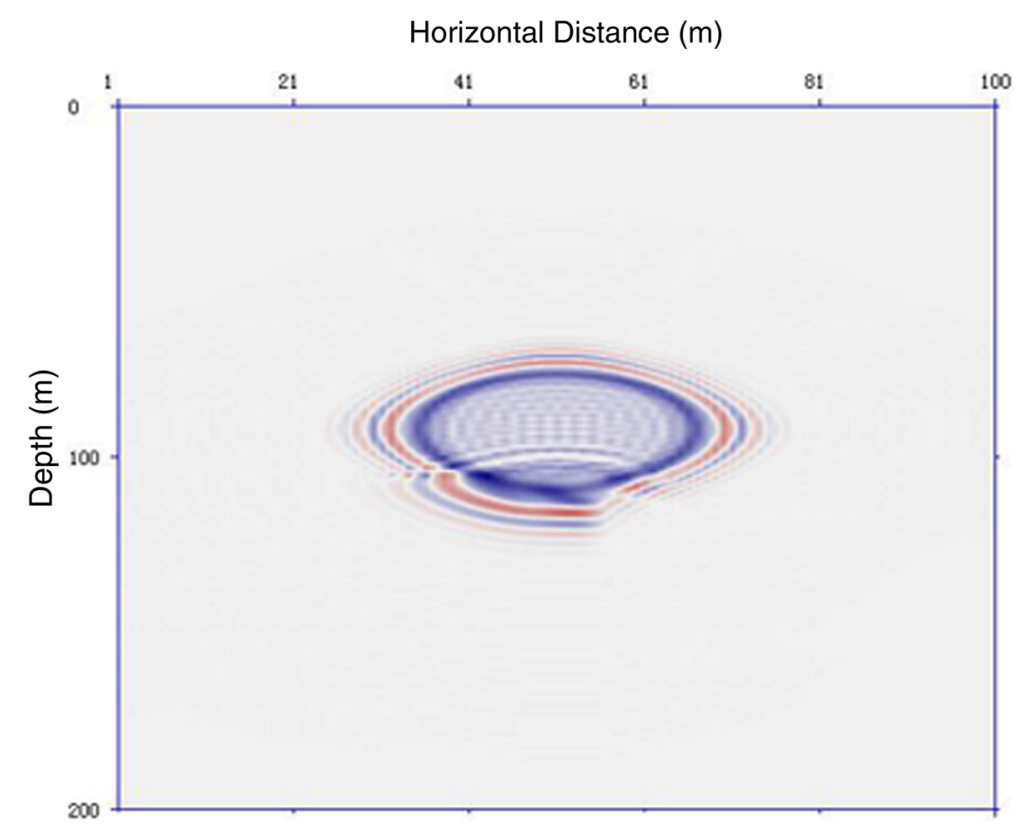

Fig. $10 t=20 \mathrm{~ms}$ snapshot of wave field of traditional method

zone are asymmetrical about the point of the velocity interface, and this law can be skillfully used in programming. At the same time, the variation of the difference points can be used as a new function for derivative formula of $Z$ direction; it is shown as follows:

$$
\frac{\partial^{2} U}{\partial z^{2}}=\frac{1}{\Delta z^{2}} \sum_{k=1}^{N} m_{k}\left(U_{i, j+n(k)}-2 U_{i, j}+U_{i, j-n(k)}\right)
$$

In which, $m_{k}$ is the difference coefficient which can be get from Taylor series, $n(k)$ is the function of the differential point number. $n(k)$ of the 10 order precision finite difference is shown in Table 1 . The difference schemes of transition zone from low-velocity layer to high-velocity layer can be derived from the same reason and the difference scheme of $X$ direction is as the same way.

\section{Results and discussion}

In general, it is too time-consuming to perform RTM imaging using a common personal computer (PC) and the efficiency is very low. In this paper, $\mathrm{PC}$ is used to

Table 3 The comparison of calculation time and memory consumption between multi-scale method and traditional method

\begin{tabular}{lll}
\hline Item & Calculation time & Memory consumption \\
\hline Traditional method & $40 \mathrm{~s}$ & $203.49 \mathrm{~kb}$ \\
Multi-scale method & $34 \mathrm{~s}$ & $188.56 \mathrm{~kb}$ \\
Efficiency promotion & $15 \%$ & $7.13 \%$ \\
\hline
\end{tabular}

perform to demonstrate the feasibility and effectiveness of this method. The computer's processor is Intel Core i5-7400, its memory is $8 \mathrm{~GB}$, and the source is the rick wavelet of $30 \mathrm{~Hz}$. The accuracy of the simulation is the 10 orders in space domain and 2 orders in time domain.

\subsection{Flat-layered model}

This is the two flat layers model; the size of the model is $100 \mathrm{~m} \times 200 \mathrm{~m}$, the velocity of the first layer is $1200 \mathrm{~m} /$ $\mathrm{s}$, the velocity of the second layer is $2400 \mathrm{~m} / \mathrm{s}$, and the velocity model is shown in Fig. 3. The two scales of the grid are employed for this model, and the design idea is that the $d x=5 m$ and $d z=2.5 m$ are used to the first layer which is the small step in $Z$ direction, and the $d x=5 m$ and $d z=5 m$ are used to the second layer which is the bigger step than the first layer in $Z$ direction; the step of the $X$ direction stays the same. The sketch map of the multi-scale design for this model is shown in Fig. 4. It should be noted that the grid of this model is from small to big, which velocity is from low to high, and the formula can be obtained by reverse recursion of Fig. 2 .

The wave field forward modeling is performed on the model, and the shot's coordinates are $50 \mathrm{~m}$ and $75 \mathrm{~m}$, the time interval is $0.5 \mathrm{~ms}$, the snapshot of wave field when $t=30 \mathrm{~ms}$ used multi-scale method is shown in Fig. 5, and in Fig. 6 is the result of traditional method. It can be seen from the two figures of the simulation results that the resolution of result based on the multi-scale method is the same as the traditional method; it can simulate the wave propagation well. 
Analysis from the perspective of comprehensive calculation efficiency, the calculation time, and the memory consumption of the multi-scale method and the traditional method is shown in Table 2. It can be seen from Table 2 that the calculation time and the memory consumption are all shortened using multi-scale method, and the comprehensive calculation efficiency is improved. From the point of view of saving time, the multi-scale method improves efficiency by $24.32 \%$.

\subsection{Step model}

There are two different velocity in the step model, and the size of the model is $100 \mathrm{~m} \times 200 \mathrm{~m}$, the velocity of the first layer is $1200 \mathrm{~m} / \mathrm{s}$, the velocity of the second layer is $2400 \mathrm{~m} / \mathrm{s}$, and the velocity model is shown in Fig. 7. It can be seen that there is a step in the model, and this model is to test and verify the applicability of multi-scale method for inflection. The coordinates of the step point are 57 and $110 \mathrm{~m}$, and 57 and $125 \mathrm{~m}$. The two scales of the grid is employed for this model, and the design idea is that the $d x=5 m$ and $d z=2.5 m$ are used to the first layer which is the small step in $Z$ direction, and the $d x=5 m$ and $d z=5 m$ are used to the second layer which is the bigger step than the first layer in $Z$ direction; the step of the $X$ direction stays the same. The sketch map of the multi-scale design for this model is shown in Fig. 8. It can be seen that the turning point of the step is in the yellow circle, and it is in the small-scale grid.
In this simulation, the coordinates of the shot are 50 and $75 \mathrm{~m}$, and the time interval is $0.5 \mathrm{~ms}$, the $t=20 \mathrm{~ms}$ snapshot of wave field used multi-scale method is shown in Fig. 9, and the result of traditional method is shown in Fig. 10. It can be seen from the result that the multi-scale method can simulate the wave propagation which agrees well with the theoretical. Analysis from the perspective of comprehensive calculation efficiency, the calculation time, and the memory consumption of the multi-scale method and the traditional method is shown in Table 3. It can be seen from Table 3 that the calculation time and the memory consumption are all shortened using multi-scale method, and the integrated computing efficiency is improved. From the point of view of saving time, the multi-scale method improves efficiency by $15 \%$. It can be found that the efficiency promotion is not the fixed value, and in this example, it is smaller than the above because of the velocity distribution of the model; the bigger the area of the high velocity, the higher the efficiency of the multi-scale method.

\subsection{SEG/salt model}

Salt model is developed by famous experts of Society of Exploration Geophysicists (SEG); the model has many highly difficult migration imaging challenges: large dip angle, many turning points, acute variety of velocity, and so on. The velocity model is shown in Fig. 11, and it can be seen that there are many large dip angles which is approaching $90^{\circ}$. The presence of salt leads to the

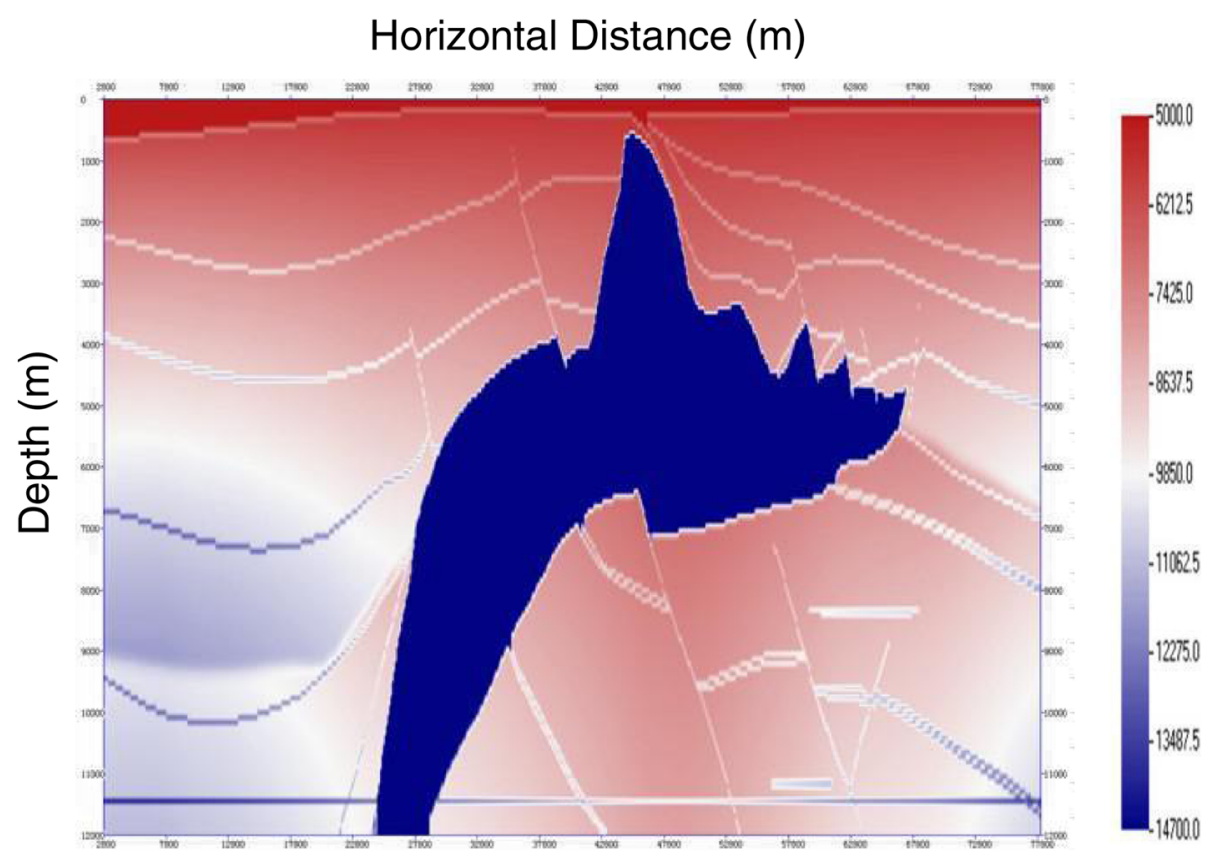

Fig. 11 The velocity of salt model 


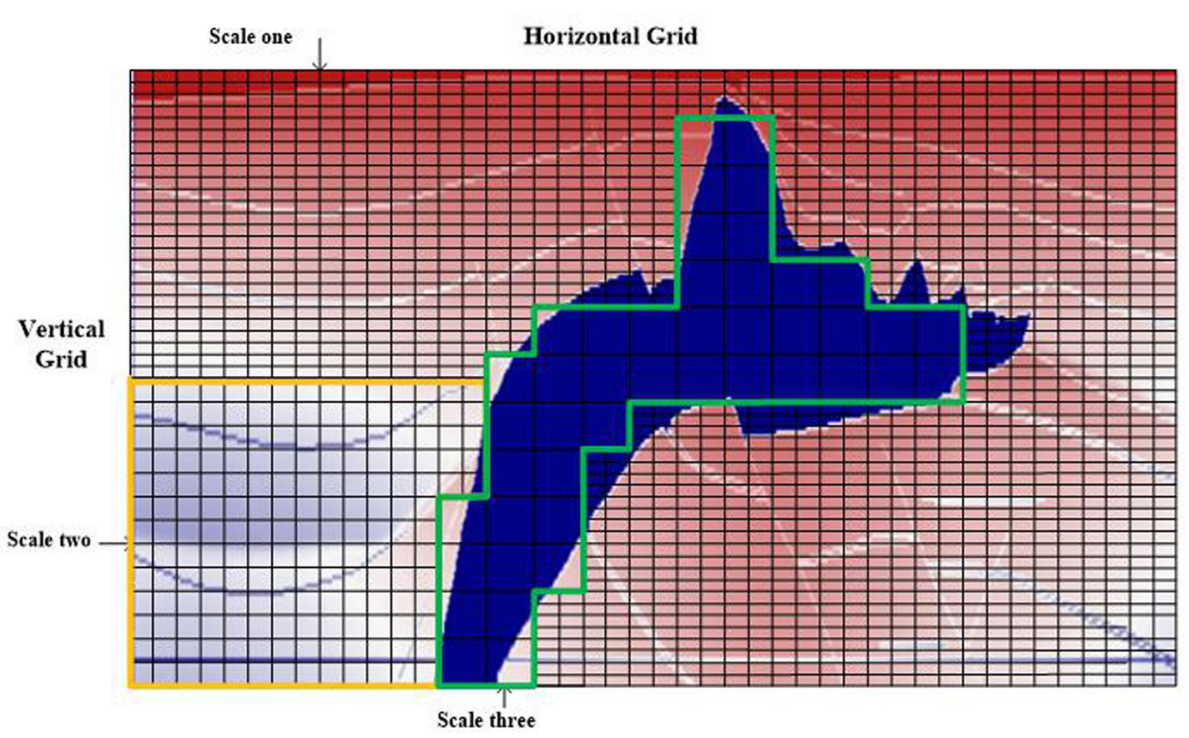

Fig. 12 The sketch map of the multi-scale design for salt model

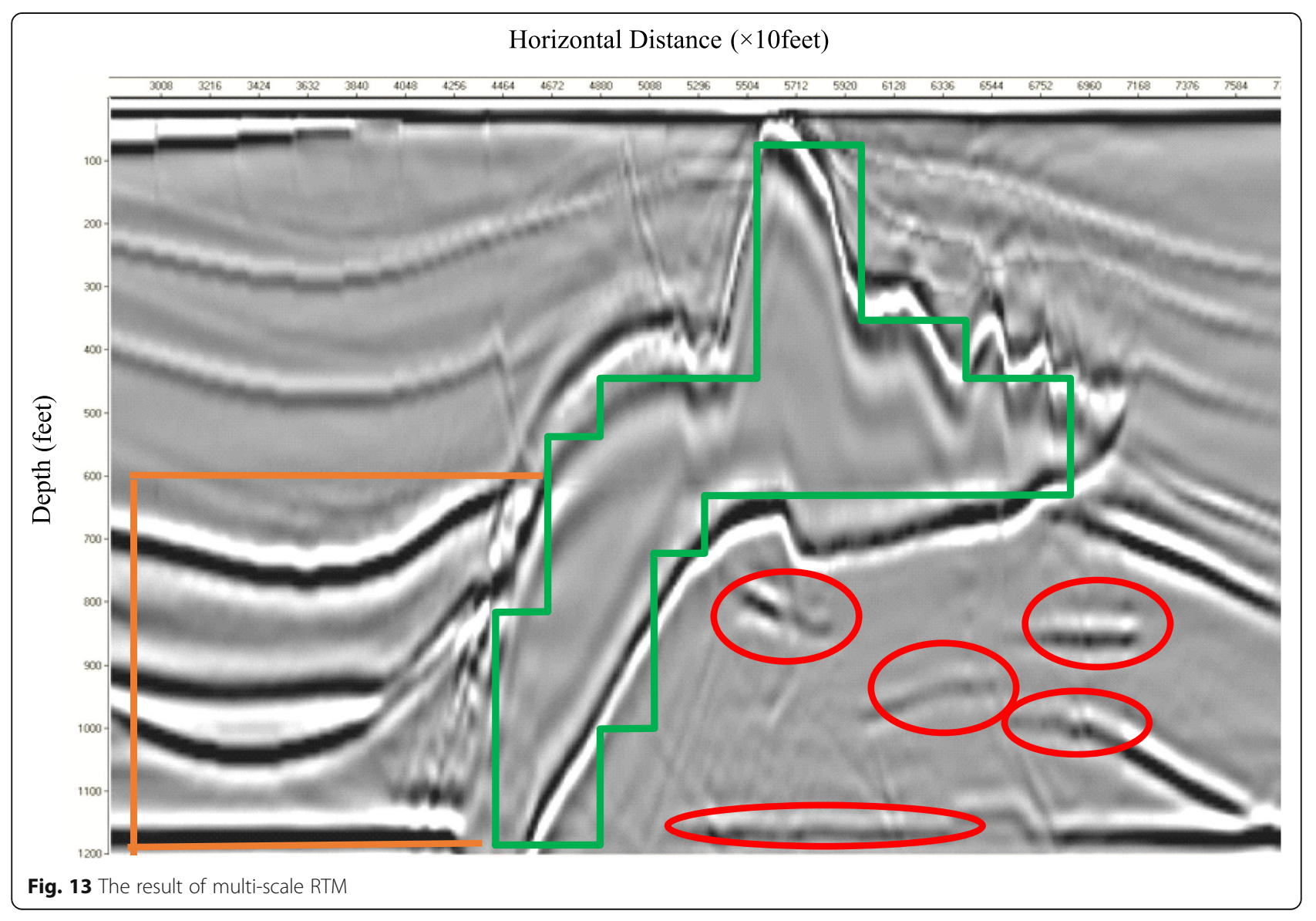




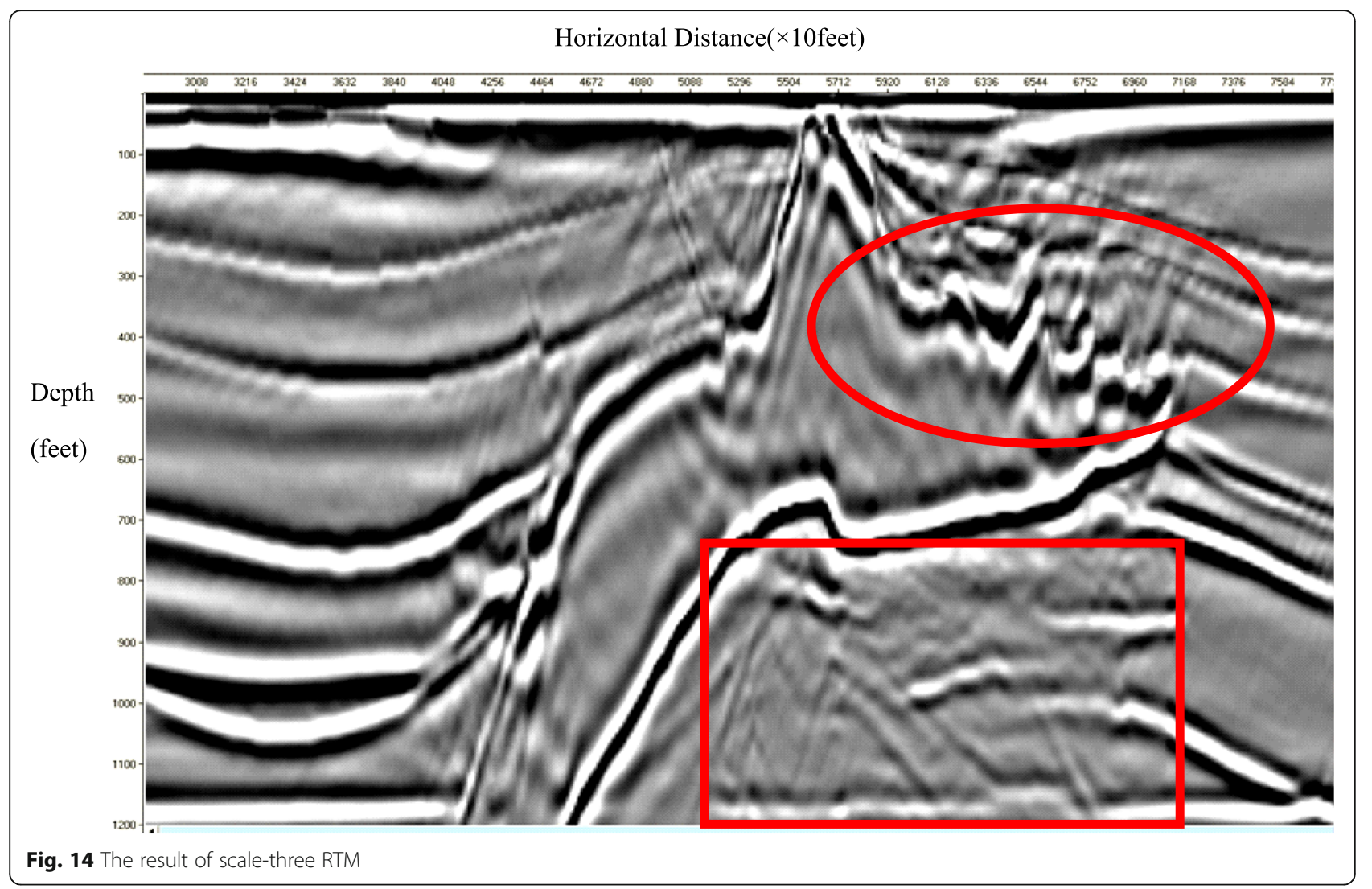

emergence of multiple inflection points in small gaps which is the difficulty for migration imaging. And the imaging of the media beneath the salt body is also the challenge to migration.

The traditional RTM uses the fix step grid of $d x=80 \mathrm{ft}$, $d z=80 \mathrm{ft}, n x=1200$, and $n z=150$. In this paper, the multi-scale RTM is employed for salt model, and three different scales are used to salt model according to the characteristics of the velocity model. The sketch map of the multi-scale design for this model is shown in Fig. 12, and it needs to be pointed out that the Fig. 12 only shows multi-scale partitioning but not the real number of the grid.

It can be seen from Fig. 12 that the most salt body which is the high-velocity area and its velocity is $14,700 \mathrm{~m} / \mathrm{s}$; this area is in the green frame which uses the grid of $d x=320 \mathrm{ft}$ and $d z=320 \mathrm{ft}$, which is the big step grid, and the edge of the salt body are almost in small step grid because the edge needs precise imaging. The area in the yellow frame uses the grid of $d x=160 \mathrm{ft}$ and $d z=160 \mathrm{ft}$; it is the medium velocity zone, and the other area of the salt model uses the original scale of $d x=80 \mathrm{ft}$ and $d z=80 \mathrm{ft}$. It needs to point that the big scale is an even multiple of small scales which is in accordance with the velocity model in this paper. In general, the relationship of the two velocities is an even number of times the scale of them is also an even multiple, if they are not an even multiple for each other, it can choose the minimum even which is bigger than real multiple. In this example, the velocity of the blue area is $14,700 \mathrm{~m} / \mathrm{s}$, and the minimum velocity is $5000 \mathrm{~m} / \mathrm{s}$ which is in the red area; the high velocity is almost three times low, so the four times is chosen for the big scale. The light blue area uses twice the scale.

The result of multi-scale RTM is shown in Fig. 13; it can be seen that the area in the green frame is imaged clearly which is the interior of the salt body that used four times the scale, the area in the yellow circle is medium velocity zone which uses two times the scale, and its imaging is clear. And the areas in the red circles are the minor faults beneath the salt body; they are also imaged well and not affected by the four times scale of the salt body. The scale-three traditional RTM result is shown in Fig. 14 and it uses the scale-three on the whole salt model; compared with Fig. 13, it can be seen that the areas in the red circle

Table 4 The comparison between multi-scale RTM and traditional RTM

\begin{tabular}{llllll}
\hline Item & \multicolumn{2}{c}{ Calculation time } & & \multicolumn{2}{c}{ Memory consumption } \\
\cline { 2 - 3 } \cline { 6 - 7 } & 1 shot & 325 shots & & shots & 325 shots \\
\hline Traditional RTM & $156.33 \mathrm{~s}$ & $13.76 \mathrm{~h}$ & & $8.21 \mathrm{M}$ & $2.60 \mathrm{G}$ \\
Multi-scale RTM & $109.87 \mathrm{~s}$ & $9.48 \mathrm{~h}$ & & $5.83 \mathrm{M}$ & $1.85 \mathrm{G}$ \\
Efficiency promotion & $29.72 \%$ & $31.10 \%$ & & $28.99 \%$ & $28.85 \%$ \\
\hline
\end{tabular}


and rectangle have lower resolution, so the precision of multi-scale RTM is better than the traditional RTM. The reason that the multi-scale RTM is very flexible to the geologic model which can use the bigger scale on higher-velocity area and the smaller scale on the lower velocity area for one geologic model, so the algorithm can get the balance between the precision and efficiency, and it can select the best one from the precision and efficiency, which is the best precision in the same efficiency and the highest efficiency in the same precision.

Table 4 is the comparison of calculation time and memory consumption between multi-scale RTM and traditional RTM. It can be observed from Table 4 that the multi-scale RTM is better than traditional RTM whether in calculation time and memory consumption, and the migration imaging is clear.

\section{Conclusions}

The paper has tested and verified that the multi-scale RTM can improve the integrated computing efficiency though the different examples, and it has improved the calculation efficiency by $25.05 \%$ average within the paper.

The multi-scale RTM is an effective method for improving computational efficiency, it has three characteristics to traditional RTM as follows: (1) it uses the big scale grid for high-velocity area to reduce the calculated amount which can improve the computational efficiency; (2) it uses scale grid for deep low speed interlayer to improve imaging accuracy; (3) it does not need to interpolate in the transition zone which can avoid the accumulation of errors caused by interpolation. In conclusion, the multi-scale RTM has the characteristics of high efficiency and precision, it lays the foundation for RTM application in practice, and the experimental results show that the algorithm is effective and reliable.

\section{Abbreviations}

FDTD: Finite difference time domain; GPU: Graphics processing unit; PC: Personal computer; RTM: Reverse time migration; SGE: Society of Exploration Geophysicists

\section{Acknowledgements}

The authors thank the editor and anonymous reviewers for their helpful comments and valuable suggestions.

\section{Funding}

The research work was supported in part by a grant from the Natural Science Foundation of China (no. 61401347), a grant from Natural Science Foundation of Shaanxi Province Department of Education of China under Grant No. 16JK1322; Xi'an Polytechnic University Doctor Scientific Research foundation of China under Grant No. BS1118.

\section{Availability of data and materials}

We can provide the data.

\section{Authors' contributions}

All authors took part in the discussion of the work described in this paper. XZ wrote the first version of the paper. LZ carried out the experiments of the paper. HG revised the paper in different version. The contributions of the proposed work are mainly in two aspects: (1) to the best of our knowledge, our work is the first one to apply the multi-scale idea for RTM imaging based on FDTD. Without any interpolation to the transitional zone, our method just deduce the wave equation though the Taylor series of mathematical method. (2) The novelty of our method attributes to the use of RTM imaging to preserve imaging accuracy while improving the integrated computing efficiency and refining the precision of the imaging area. All authors read and approved the final manuscript.

\section{Authors' information}

Xiaodan Zhang received the B.S. degree in communication engineering from Xi 'an Communication College, Xi'an, China, in 2004, the MS degree in circuit and system from Xi'an University of Technology, Xi'an, China, in 2008, and the Ph.D degree in Microelectronics and Solid-State Electronics from Xi'an University of Technology, Xi'an, China, in 2012. In 2011, She joined Xi'an Polytechnic University, Xi'an, China. From 2011 to present, she was a lecturer in the College of Electronic and Information at Xi'an Polytechnic University. Since September 2017, she joined Xi 'an Jiaotong University as a young backbone visiting scholar. Her research interests include migration imaging, imaging signal processing, and wavelet analysis.

Lei Zhu received his B. S. and M. S. degrees form communication engineering, Xi'an Polytechnic University in 2002 and 2005, respectively, and Ph.D degree in signal and information processing in Xidian University in 2012. He is a Professor at the college of Electronic and Information, Xi'an University of Technology. His current research interests include SAR image processing, computer vision.

Hua Guo received his B. S. degree in applied physics frOm Xidian University in 2004, and M. S. degrees in radio physics from Xidian University in 2007, and the Ph.D. degree in electromagnetic field and microwave technology from Northwestern Polytechnical University in 2015. He is a lecturer in the college of electronic and information at Xi'an Polytechnic University. His current research interests include array signal processing, image processing, and machine learning.

\section{Competing interests}

There are no potential competing interests in our paper.

\section{Publisher's Note}

Springer Nature remains neutral with regard to jurisdictional claims in published maps and institutional affiliations.

Received: 11 April 2018 Accepted: 13 June 2018

Published online: 06 July 2018

References

1. $Z \mathrm{Li}, \mathrm{X} \mathrm{Li}$, J Xiao, B Hu, Amplitude-preserved reverse time migration with Gaussian beam based on crosscorrelation imaging conditions. Comput Tech Geophys Geochem Explor 39, 354-358 (2017)

2. E Baysal, DD Kosloff, JWC Sherwood, Reverse time migration. Geophysics 48, 1514-1524 (1983)

3. H Guan, E Dussaud, B Denel, P Williamson, in SEG 81st Annual International Meeting: September 18-23, 2011; San Antonio, USA. Techniques for an efficient implementation of RTM in TTI media (2011), pp. 3393-3397

4. W Dai, P Fowler, GT Schuster, Multisource least-squares reverse-time migration. Geophys. Prospect. 60, 681-695 (2012)

5. H Wen, D Zhang, X Zheng, S Fan, W Wang, Propagation characteristics of electromagnetic wave based on FDTD in coal. J. China Coal Soc. 42, 29592967 (2017)

6. $\quad Y$ Zhang, L Duang, Y Xie, in SEG 83rd Annual International Meeting: September 22-27, 2013; Houston, USA. A stable and practical implementation of least-squares reverse time migration (2013), pp. 3716-3720

7. Y Liu, WW Symes, Z Li, in SEG 83rd Annual International Meeting: September 22-27, 2013; Houston, USA. Multisource least-squares extended reverse-time migration with preconditioning guided gradient method (2013), p. 1251

8. DC Del Rey Fernandez, JE Hicken, DW Zingg, Review of summation-by-parts operators with simultaneous approximation terms for the numerical solution of partial differential equations. J. Sci. Comput. 95, 171-196 (2014)

9. B Sjögreen, High order finite difference and finite volume methods for advection on the sphere. J. Sci. Comput. 51, 703-732 (2014)

10. B Sjögreen, NA Petersson, A fourth order accurate finite difference scheme for the elastic wave equation in second order formulation. J. Sci. Comput. 52, 17-48 (2012) 
11. Y Rao, YH Wang, Seismic waveform simulation with pseudoorthogonal grids for irregular topographic models. Geophys. J. Int. 194, 1778-1788 (2013)

12. B LI, HW LIU, GF LIU, Computational strategy of seismic pre-stack reverse time migration on CPU/GPU. Chin. J. Geophys. 53, 2938-2943 (2010)

13. WG Wang, SJ Xiong, HN Xu, RY Qian, TTI media anisotropic pseudospectral method inverse time migration. Pet Geophys Explor 47, 566$572+682+513(2012)$

14. JE Kozdon, EM Dunham, J Nordstrom, Simulation of dynamic earthquake ruptures in complex geometries using high-order finite difference methods. J. Sci. Comput. 55, 92-124 (2013)

15. JP Huang, Q Zhang, K Zhang, ZC Li, YB Yue, ML Yuan, Green function gaussian beam inverse time deviation. Pet Geophys Explor 49, 101-106+303 (2014)

16. B Wang, J Gao, W Cheng, $\mathrm{H}$ Zhang, Efficient boundary storages strategies for seismic inverse time migration. Chin. J. Geophys. 55, 2412-2421 (2012)

17. H Zhao, J Gao, Y Ma, An absorbing boundary algorithm for diffusive-viscous wave equation. J. Xi'an Jiaotong Univ. 46, 112-118 (2012)

18. W Meng, L-Y Fu, Seismic wavefield simulation by a modified finite element method with a perfectly matched layer absorbing boundary. J. Geophys. Eng. 14, 852 (2017)

19. L Na, Multi-source least-squares inverse time migration based on Huber norm. Pet Geophys Explor 52, 941-947+878 (2017)

20. Y Wang, H Zhou, S Yuan, Y Ye, A fourth order accuracy summation-by-parts finite difference scheme for acoustic reverse time migration in boundaryconforming grids. J. Appl. Geophys. 136, 498-512 (2017)

\section{Submit your manuscript to a SpringerOpen ${ }^{\bullet}$} journal and benefit from:

- Convenient online submission

- Rigorous peer review

- Open access: articles freely available online

- High visibility within the field

- Retaining the copyright to your article

Submit your next manuscript at $\gg$ springeropen.com 